\title{
ERRATUM
}

\section{Waldenstrom macroglobulinemia: prognosis and management}

A Oza and SV Rajkumar

Blood Cancer Journal (2016) 6, e391; doi:10.1038/bcj.2015.42; published online 5 February 2016

Correction to: Blood Cancer Journal (2015) 5, e296; doi:10.1038/ bcj.2015.28; Published online 27 March 2015

After the publication of this paper, it was noticed that the e-number for this paper is incorrect. It will now be changed from e296 to e394.
The publishers would like to apologise for any inconvenience this may have caused. 